\title{
A phase II study evaluating the use of concurrent mitomycin $C$ and capecitabine in patients with advanced unresectable pseudomyxoma peritonei
}

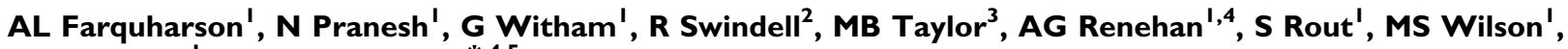 \\ ST O'Dwyer' and MP Saunders*,4,5
}

'Peritoneal Tumour Service, Department of Surgery, Christie Hospital NHS Foundation Trust, Manchester, UK; ${ }^{2}$ Department of Medical Statistics, Christie Hospital NHS Foundation Trust, Manchester, UK; ${ }^{3}$ Department of Radiology, Christie Hospital NHS Foundation Trust, Manchester, UK; ${ }^{4}$ School of Cancer and Imaging Sciences, University of Manchester, Manchester, UK; ${ }^{5}$ Department of Clinical Oncology, Christie Hospital NHS Foundation Trust,

Manchester, UK

Pseudomyxoma peritonei (PMP) is a rare neoplastic process characterised by progressive intra-abdominal dissemination of mucinous tumour, and generally considered resistant to systemic chemotherapy. A phase II study in patients with advanced unresectable PMP was undertaken to evaluate the combination of systemic concurrent mitomycin $C\left(7 \mathrm{mg} \mathrm{m}^{-2}\right.$ i.v. on day I) and capecitabine ( I $250 \mathrm{mg} \mathrm{m}^{-2}$ b.d. on days I - |4) in a 3-weekly cycle (MCap). Response was determined by semiquantitative assessment of disease volume on serial computed tomographic (CT) scans and serum tumour marker (CEA, CAI25, CAI9-9) changes at 12 weeks. Between 2003 and 2006, 40 patients were recruited through a national centre for the treatment of peritoneal surface tumours. At baseline, 23 patients had progressive disease and 17 had stable disease. Of 39 assessable patients, 15 (38\%, 95\% confidence intervals (Cls): $25,54 \%$ ) benefited from chemotherapy in the form of either reductions in mucinous deposition or stabilisation of progressive pretreatment disease determined on CT scan. Notably, two patients, originally considered unresectable, following MCap and re-staging underwent potentially curative cytoreductive surgery. Grade 3/4 toxicity rates were low (6\%, 95\% Cls: 4, 9\%). Twenty out of 29 assessed patients (69\%, 95\% Cls: 5I, 83\%) felt that their Global Health Status improved during chemotherapy. This is the first trial to demonstrate an apparent benefit of systemic chemotherapy in patients with advanced unresectable PMP.

British Journal of Cancer (2008) 99, 59I-596. doi: I0.1038/sj.bjc.6604522 www.bjcancer.com

Published online 5 August 2008

(c) 2008 Cancer Research UK

Keywords: pseudomyxoma peritonei; chemotherapy; capecitabine; mitomycin C

Pseudomyxoma peritonei (PMP) is a rare neoplastic process (approximately 1-2 per million per year), arising in the majority of cases from the appendix, defined by disseminated peritoneal mucinous tumour deposition and progressive accumulation of mucinous ascites (Renehan et al, 2008). Since mid 1990s, aggressive cytoreductive surgery with removal of all visible macroscopic disease, followed by hyperthermic intraperitoneal chemotherapy (HIPEC) - often referred to as the 'Sugarbaker' procedure - has been promulgated as standard care (Sugarbaker, 2006). However, complete cytoreduction is possible in only one-third of cases at presentation (Rout et al, 2008). Disease features that prohibit complete cytoreduction include gastric encasement, diffuse small bowel mesenteric involvement and fistulation (Sugarbaker and Chang, 1999). For such advanced unresectable cases, debulking surgery may offer symptomatic relief, but is associated with

\footnotetext{
*Correspondence: Dr MP Saunders, Department of Clinical Oncology, Christie Hospital NHS Trust, Wilmslow Road, Manchester M20 4BX, UK; E-mail: mark.saunders@christie.nhs.uk

Received 26 February 2008; revised 23 June 2008; accepted 30 June 2008; published online 5 August 2008
}

considerable morbidity and mortality (Murphy et al, 2007; Smeenk et al, 2007).

Conventionally, PMP is considered resistant to systemic chemotherapy, and, to date, there are no published chemotherapy trials in the setting of advanced unresectable disease. However, a recent report described a patient with PMP responding to capecitabine - an oral fluoropyrimidine (Levitz et al, 2004). Given this, and the following rationales: (i) mitomycin $\mathrm{C}$ (MMC) and 5-fluorouracil (5-FU) are administered as HIPEC in the management of PMP (Witkamp et al, 2001; Sugarbaker, 2006); (ii) MMC is an established antitumour agent against gastrointestinal cancers (Crooke and Bradner, 1976; Ozawa et al, 1988); and (iii) oral capecitabine has equivalence effectiveness to bolus intravenous 5-FU/folinic acid in the treatment of metastatic colorectal cancer (Hoff et al, 2001; Van Cutsem et al, 2001), but with better patient acceptance (Borner et al, 2002), we hypothesized that a combination of concurrent MMC and capecitabine (MCap) would benefit patients with advanced PMP. Furthermore, this regimen is effective in advanced colorectal cancer (Rao et al, 2004).

The aim of this study was to undertake a phase II study to evaluate tumour response, survival, toxicity and quality of life in patients undergoing MCap chemotherapy for advanced unresectable PMP. 


\section{MATERIALS AND METHODS}

\section{Patients}

Between April 2003 and December 2006, patients referred with histologically confirmed PMP to the Peritoneal Tumour Service of Christie Hospital NHS Foundation Trust (Manchester, UK), one of the two national centralised centres in the UK, and considered unresectable by a dedicated PMP multidisciplinary team meeting, were considered for this trial. Unresectability was determined by predefined criteria on oral contrast computed tomography (CT) scan, namely, gastric encasement, extensive small bowel mesenteric involvement and/or fistulating disease, supplemented wherever possible by pre-referral operative records and/or intraoperative photographs. Histological classification was described by Ronnett et al (1997),-namely, disseminated peritoneal adenomucinosis (DPAM), peritoneal mucinous carcinomatosis (PMCA) and PMCA with intermediate or discordant features (PMCA-I/D). The trial was conducted with local ethical approval, in accordance with accepted standards of good clinical practice, and in agreement with the Declaration of Helsinki.

\section{Inclusion and exclusion criteria}

The inclusion criteria were as follows: age $\geqslant 18$ years, World Health Organisation performance status $0-2$ and life expectancy $>3$ months. All patients were required to have adequate haematological function (neutrophil count $\geqslant 1.5 \times 10^{9}$ per $l$ and platelet count $\geqslant 150 \times 10^{9}$ per 1 ), hepatobiliary function (serum bilirubin $\leqslant 1.5 \times$ upper limit of normal $(\mathrm{ULN})$; ALP $\leqslant 5 \times \mathrm{ULN}$; transaminase (AST or ALT) $\leqslant 3 \times$ ULN) and renal function (estimated Cockcroft clearance $\geqslant 50 \mathrm{ml} \mathrm{min}^{-1}$ ). Advice about contraceptive precautions was given, and for women of childbearing potential, a negative pregnancy test was required.

The exclusion criteria were as follows: previous MMC or 5-FU-based systemic chemotherapy; concurrent uncontrolled medical illness, including cardiac disease, and previous or concurrent malignant disease; evidence of bowel obstruction; chronic diarrhoea; or if women were pregnant or lactating. Patients who had experienced life-threatening toxicities with fluoropyrimidine treatment or had any condition that might affect the absorption of capecitabine were also excluded. Patients on warfarin anticoagulation were changed to low-molecular-weight heparin (enoxaparin sodium) at the appropriate therapeutic dose.

\section{Treatment}

The MCap regimen consisted of MMC $7 \mathrm{mg} \mathrm{m}^{-2}$ intravenous injection on day 1 and capecitabine $1250 \mathrm{mg} \mathrm{m}^{-2}$ twice daily on days 1-14, with a break in treatment on days 15-21. The next cycle consisted solely of capecitabine $1250 \mathrm{mg} \mathrm{m}^{-2}$ twice daily on days $1-14$, with a break in treatment on days 15-21. These 3-week cycles were alternated so that eight cycles were given in total (four of each) (Figure 1). Prophylactic anti-emetics (ondansetron $8 \mathrm{mg}$ ) were administered intravenously along with MMC. Patients were treated for at least 12 weeks $(4 \times 3$-weekly cycles $)$ prior to response evaluation.

\section{Assessment of tumour response and toxicity}

Patients were assessed at baseline and every 3 weeks and the following parameters recorded: performance status, weight, abdominal girth and toxicity scored using the National Cancer Institute's Common Terminology Criteria for Adverse Events version 3.0 (NCI, 2004). The following serum tumour markers were determined: carcinoembryonic antigen (CEA), cancer antigen 125 (CA125) and cancer antigen 19-9 (CA19-9).

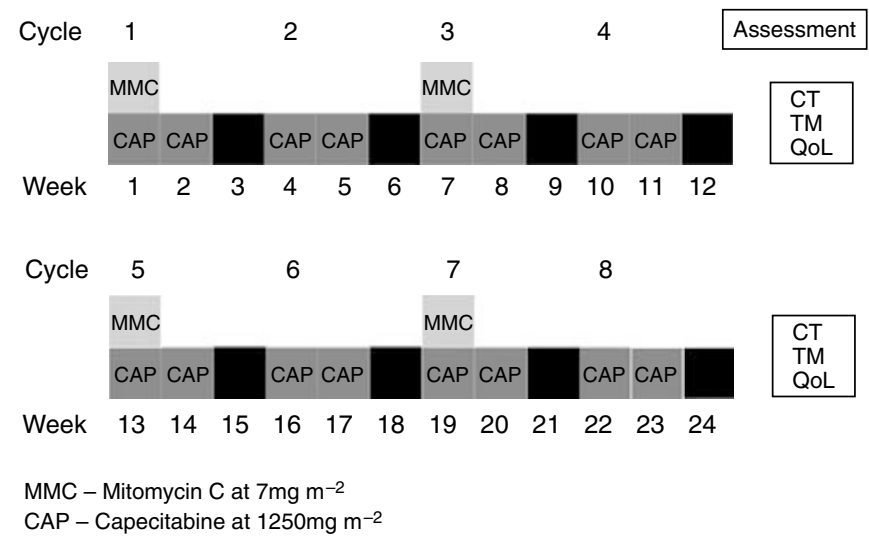

Figure I The MCap chemotherapy regimen. CT, CT scan at baseline and then 3-monthly; TM, tumour markers: CAI25, CAI9.9, CEA; QoL: quality of life data collected at each cycle.

A CT (axial computed tomography) scan was performed at baseline, and at the end of cycles 4 (12 weeks) and 8 (24 weeks). Standard CT scan RECIST criteria for the assessment of disease response were not applicable, as disease characteristics, such as cystic areas and ascites, are not included in these criteria (Padhani and Ollivier, 2001). Instead, using parallel matched computer monitors of serial CT scans, tumour response was determined semiquantitatively as either stable, reduced or progressed.

Chemotherapy was administered unless a criterion for study discontinuation was met. Treatment was stopped at the request of the patient for any reason, or if, in the opinion of the investigator, it was in the patient's best interest to do so. At each review, a score of toxicity criteria was made according to NCI-CTCAE documents. Patients were treated only if all of these toxicity criteria were grade 1 or less. Patients with persistent grade 2 symptoms were deferred until their symptoms had improved to at least grade 1 . If patients experienced grade 3 or 4 toxicity, then chemotherapy was delayed until their symptoms had resolved to grade 1 or better. Further doses of capecitabine and MMC were then reduced by $20 \%$ and this was continued for the rest of the course.

\section{Assessment of quality of life}

Quality of life was assessed using the European Organization for Research and Treatment of Cancer (EORTC) questionnaires QLQC30 (version 3) (Aaronson et al, 1993) and QLQ-CR38 (version 3) (Sprangers et al, 1999) at baseline, at 6 weeks and then every 12 weeks during the chemotherapy course.

\section{Statistical analysis}

Data were collected prospectively and analysed using SPSS version $14.0^{\circledR}$ (Superior Performing Software Systems, Chicago, IL, USA). $95 \%$ confidence intervals (CIs) for single proportions were estimated using the Wilson score method without continuity correction (Newcombe, 1998). The Wilcoxon matched-pairs signed-rank test was used to compare pre- and post-treatment changes in tumour markers and quality of life data from the EORTC questionnaires QLQ C30 and CR38. Survival estimates were calculated by using the Kaplan-Meier method. Statistical significance was taken as $P \leqslant 0.05$.

As there was no precedent, a response rate of at least $20 \%$ was considered to be acceptable in this study. Therefore, 14 patients were treated in the first instance and the study was extended on the response measured in that group. Only patients receiving the first 12 weeks of treatment were included in the assessment of the objective response rate. As more than 1 out of the first 14 patients 
Table I Baseline patient characteristics

\begin{tabular}{lc}
\hline & $\mathbf{n}^{\mathbf{a}} \mathbf{( \% )}$ \\
\hline Patients & 40 \\
Median age (range), years & $59(32-77)$ \\
Males/females & $12: 28$ \\
Histological group & \\
DPAM & \\
PMCA-l/D & $27(68)$ \\
PMCA & $10(25)$ \\
Previous laparotomies & 3 \\
No previous laparotomies & \\
I & 1 \\
2 & $12(30)$ \\
$\geqslant 3$ & 5 \\
WHO performance status & \\
0 & \\
1 & $22(55)$ \\
2 & $36(90)$ \\
\hline
\end{tabular}

DPAM = disseminated peritoneal adenomucinosis; PMCA $=$ peritoneal mucinous carcinomatosis; PMCA-I/D = PMCA with intermediate or discordant features. a Number of patients unless otherwise stated.

responded, the minimum number of patients required to obtain a measurable response was 27 and the maximum 40 in order to give an estimate of the true response rate to a s.d. of $7.5 \%$.

\section{RESULTS}

Forty patients were recruited to the trial. Baseline characteristics are shown in Table 1. Over one-half of the participants were female; all but one patient had undergone laparotomy elsewhere prior to referral; and two-thirds were histologically classified as DPAM. Two patients underwent laparotomy at our centre with a view to complete cytoreduction but were considered unresectable at operation and referred to the trial.

\section{Tumour response and survival}

The flow diagram of disease status at baseline and subsequent responses to treatment are shown in Figure 2. Out of the 40 patients who started treatment with MCap chemotherapy, $23(58 \%)$ had progressive disease and $17(42 \%)$ had stable disease at baseline measurements (Figure 2).

Out of the 23 patients with progressive disease prior to trial entry, 19 patients received 3 months of treatment and 15 completed a full course of chemotherapy over 6 months. One patient had a fit during the first cycle and had no further treatment and was therefore not assessable for response. Three patients (14\%) responded to the treatment with a reduction in the volume of mucus, and one of these patients also had a reduction in the volume of the solid component of their disease. After 6 months of chemotherapy, these patients had a sustained reduction in disease and nine patients $(41 \%)$ were found to have stable disease. These patients had progressive disease prior to chemotherapy (Figure 2).

Prior to treatment, two patients had mucous discharge of approximately $50 \mathrm{ml} \mathrm{day}{ }^{-1}$ from cutaneous fistula/sinuses. In one patient, the discharge settled completely with the cutaneous opening healing after 3 months of treatment. Another patient had two sinuses at the start of the treatment. After chemotherapy, one of the sinuses was dry and discharge from the other reduced from $50 \mathrm{mlday}^{-1}$ to approximately $20 \mathrm{ml} \mathrm{day}^{-1}$. There was also a change in the consistency of the discharge from mucoid to serous (Figure 3).

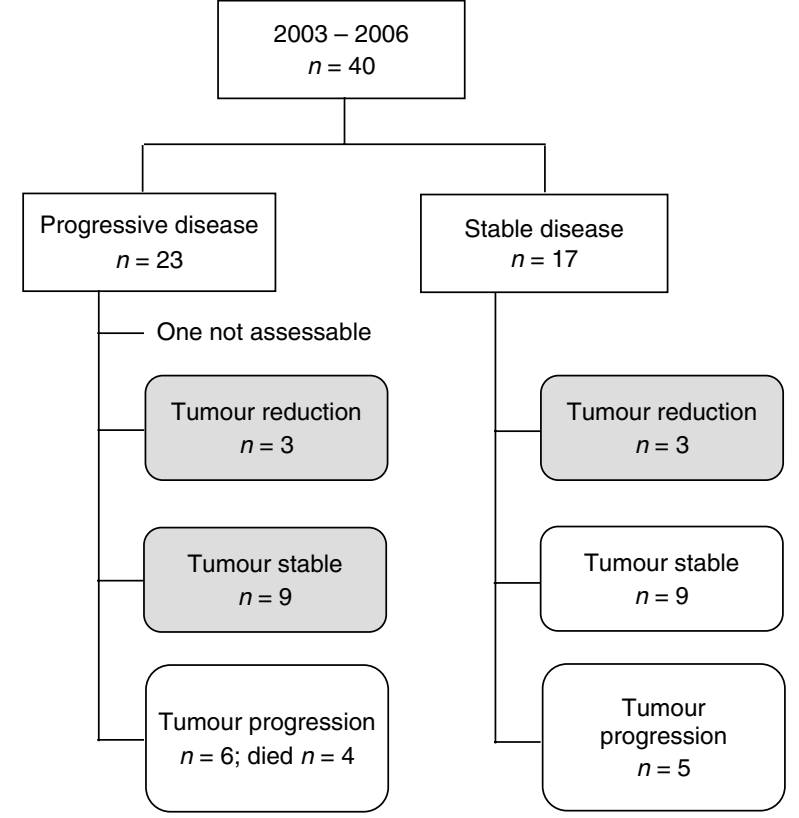

Figure 2 Flow diagram of treatment response. Response determined by CT scan during chemotherapy at 12 and 24 weeks and then confirmed at 6 months.

Out of the 17 patients with stable disease prior to trial entry, 16 patients completed a full course of treatment. At 3 months, all 17 patients were receiving chemotherapy and were therefore assessable. Three patients (18\%) responded to treatment with a reduction in the volume of mucus, which was maintained at 6 months. At this time point, nine patients $(53 \%)$ were found to have stable disease and five (29\%) were found to have progressive disease (Figure 2).

Overall, out of the 39 assessable patients, 15 (38\%) benefited from the chemotherapy regimen in terms of a reduction in mucus (with or without a solid component) or development of stability when known to be progressing prior to treatment (Figure 2). Patients who had stable disease prior to starting treatment and had maintained stable disease during chemotherapy were considered to have received no added benefit from this therapy.

With a median follow up of 17 months (range 3.3-34.0), the 1 -year and 2-year tumour-related survival rates for the 40 patients were $84 \%$ and $61 \%$, respectively (Figure 4 ).

\section{Tumour marker response}

At baseline measurement, at least one serum tumour marker was raised in all patients and all three tumour markers were raised in 13 patients (33\%, 95\% CIs: $20,48 \%)$. For those patients who completed a full chemotherapy course, there were reductions in the tumour marker levels by more than 50\% for CEA in 11 patients; CA125 in 7 patients and CA19-9 in 6 patients. Twenty patients $(51 \%, 95 \%$ CIs: $36,66 \%)$ had a reduction in one or more tumour markers by more than $50 \%$ with chemotherapy. Overall, there were statistically significant reductions in the concentrations of CEA $(P=0.001)$ and CA125 $(P=0.002)$, but not CA19-9, between pre- and post-trial values (Figure 5). However, there were no differences in pre- and post-chemotherapy tumour marker changes when stratified by tumour response, histological type and whether progressive or stable disease at baseline. Weight, abdominal girth and performance status, which were measured at each cycle, did not alter significantly, and were not related with patient's response to treatment. 

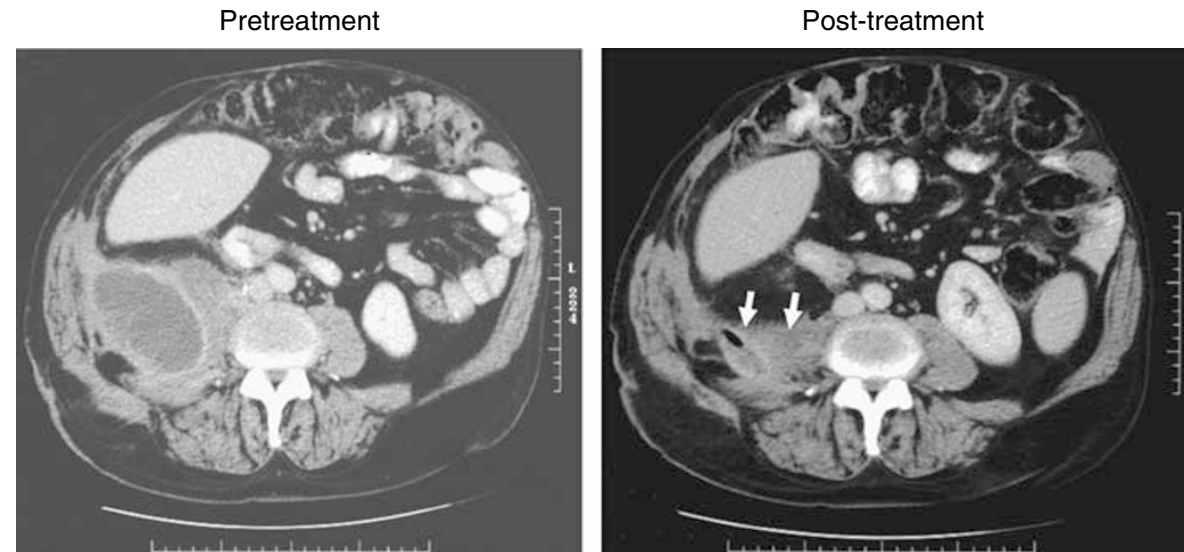

Figure 3 Computed tomographic scan of the abdomen at baseline and after the full treatment in a patient with initially progressive disease that shows a reduction in size of the retroperitoneal collection (arrows).

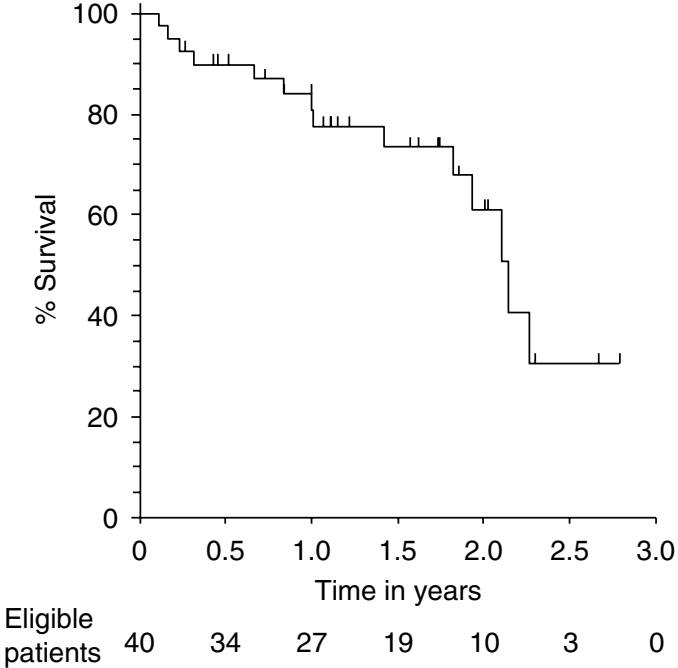

Figure 4 Survival curve for patients receiving MCap chemotherapy using Kaplan-Meier plot.

\section{Toxicity}

There were no treatment-related mortalities; four patients (all in the progressive disease group at baseline) died early during their treatment due to disease progression; three of them had either PMCA-I/D or PMCA histological subtypes. Thirty-one patients (78\%, 95\% CIs: 63, 88\%) completed 24 weeks of MCap chemotherapy. One patient suffered an epileptic fit during the first cycle and had no further treatment. Three patients stopped chemotherapy after the interim scans showed progressive disease; one patient stopped before the final cycle due to grade 3 toxicity. Grade 3 toxicities occurred in 12 out of 277 cycles; grade 4 toxicities in 4 out of 277 cycles - an overall rate of $6 \%$ (95\% CIs: $4,9 \%)$. All these toxicities were the hand and foot syndrome (Table 2).

\section{Repeat MCap chemotherapy}

Despite disease progression, 17 patients had a repeat MCap chemotherapy. Of these, $7 \%(41,95 \%$ CIs: $22,64 \%)$ achieved disease stabilisation. The maximum amount of MMC given was 6 doses of $7 \mathrm{mg} \mathrm{m}^{-2}$ ( $42 \mathrm{mg} \mathrm{m}^{-2}$ total) - no cumulative MMC toxicity was evident. After this, patients were only treated with capecitabine at the same dose. Notably, two patients, originally considered

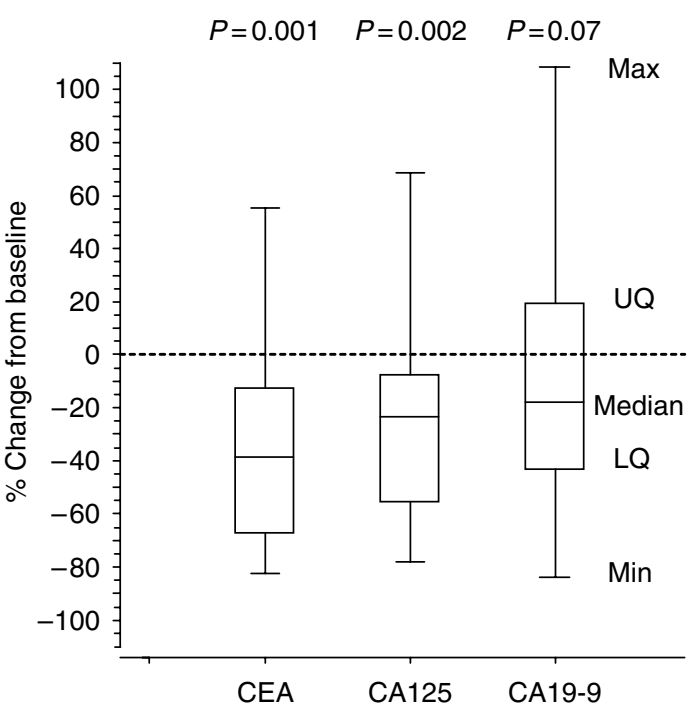

\begin{tabular}{|cccc|}
\hline $\begin{array}{c}\text { No. of patients with } \\
\text { elevated baseline levels }\end{array}$ & 40 & 25 & 26 \\
\hline Median baseline (range) & $\begin{array}{c}25 \\
(3-591)\end{array}$ & $\begin{array}{c}46 \\
(3-280)\end{array}$ & $\begin{array}{c}115 \\
(5-24000)\end{array}$ \\
\hline Median \% change (range) & $\begin{array}{c}-38 \\
(-82 \text { to } 56)\end{array}$ & $\begin{array}{c}-24 \\
-78 \text { to 69) }\end{array}$ & $\begin{array}{c}-84 \text { to 108) } \\
-18\end{array}$ \\
\hline $\begin{array}{c}\text { No. of patients }>50 \% \\
\text { marker reduction }\end{array}$ & 11 & 7 & 6 \\
\hline
\end{tabular}

Figure 5 Percentage change in tumour marker level from baseline to post-treatment. Analysis limited to patients who completed a full course. CEA, carcinoembryonic antigen (normal range $<3 \mu \mathrm{gI} \mathrm{I}^{-1}$ ); CAI25, cancer antigen 125 (normal range $<30 \mathrm{U} \mathrm{ml}^{-1}$ ); CAI9-9, cancer antigen (normal range $\left.<31 \mathrm{U} \mathrm{ml}^{-1}\right)$.

unresectable, following MCap and re-staging underwent potentially curative cytoreductive surgery.

\section{Quality of life}

Overall, quality-of-life parameters remained stable, with only the Global Health Status and chemotherapy-related side effects showing some alteration with treatment. The Global Health Status (QL2) provides an insight into a patients overall feeling about their 
Table 2 Number and grade of toxicity events at each cycle during course

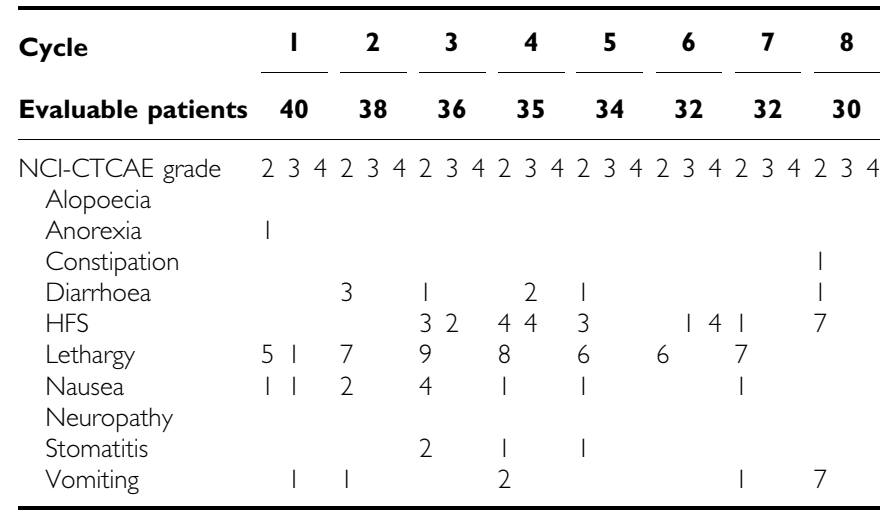

HFS = hand and foot syndrome; NCl-CTCAE v3.0 = National Cancer Institute Common Toxicity Criteria for adverse events.

well being. In this regard, out of the 15 patients with initially progressive disease prior to chemotherapy who completed a full course, $10(67 \%)$ felt that their Global Health Status was the same or better at the end of the course, but 4 felt that it was worse (1 patient did not complete the form). Out of the 16 patients with initially stable disease prior to chemotherapy who completed a full course, $10(63 \%)$ felt that their Global Health Status was the same or better at the end of the course, but 5 felt that it was worse (1 patient did not complete the form). Analysis of data from each section of the questionnaire showed that the only statistically significant reduction in a specific area of quality of life was chemotherapy-related side effects $(P=0.005)$. There was no statistically significant difference in change in quality of life during the chemotherapy course stratified by initial disease status (stable $v s$ progressive).

\section{DISCUSSION}

This is the first evaluation of systemic chemotherapy in patients with advanced unresectable PMP. Within the limitation of a nonrandomised design, the data show that over one-third of patients appear to benefit from treatment with MCap chemotherapy without high rates of severe toxicity or reduction in quality of life.

A potential limitation of the study was the failure to quantify disease response using volume-based criteria. The role of CT scanning is well outlined for initial diagnosis. Early disease is characterised by an appendiceal tumour, and deposits predominantly on the diaphragmatic surface, around the liver and spleen and within the pouch of Douglas; as the disease progresses, deposits around the liver and spleen show scalloping and compress the adjacent organs, particularly the stomach (Sulkin et al, 2002). On contrast enhanced CT, PMP deposits are predominantly of low density, but may show septa and solid elements, likely to represent mucinous tumour deposits, encysted collections of mucin and fibrosis. Because of the diffuse nature of the disease, measurement of PMP disease volume is challenging. The RECIST criteria are not applicable, as these specifically exclude ascites and cystic areas as measurable lesions (Padhani and Ollivier, 2001). Changes in volume of disease within the peritoneal cavity may be assessed in some cases by measurement of relatively well-defined deposits (encysted mucin) or by measurement of the radial diameter of deposits over the surface of the liver or spleen. However, where there are diffuse deposits, volume of disease cannot be meaningfully assessed using unidimensional or bidimensional measurements. Moreover, apparent changes in the burden of intraperitoneal disease may reflect changes in the volume of mucinous ascites and not of solid tumour. The baseline scan and all subsequent scans were carried out at Christie Hospital using the same protocol. All scans were reported by radiologists who are experienced in the radiological evaluation of PMP.

In an effort to overcome these potential limitations, CT assessment of treatment was approached systematically using three criteria: (i) overall assessment of the disease volume including mucin (supported by measurement of discrete deposits where possible); (ii) recording of new disease sites and (iii) the extent of compressive effects of disease on intraperitoneal organs, for example, degree of scalloping of the liver and spleen, compression of the stomach and strictures of the small or large bowel. Decreases in volumes of mucin alone were not recorded as responses.

The present study has a number of advantages. First, data on treatment responses, survival, toxicity and quality of life were collected prospectively. Second, uniform criteria were used to report histological classifications and radiological responses. Third, the study was set within the framework of a dedicated Peritoneal Tumour Multidisciplinary meeting (Rout et al, 2008), which assesses resectability and directs patients along appropriate clinical pathways. This achievement was brought about through the establishment of two centralised national treatment services Christie Hospital NHS Foundation Trust (Manchester, UK) and North Hampshire Hospitals NHS Foundation Trust (Basingstoke, UK) (Moran, 2006)-commissioned by the UK National Health Service (NHS) National Commissioning Group for Highly Specialised Services. This funding system circumnavigates many logistic problems of undertaking a trial in a rare tumour.

We explored whether serum tumour markers, namely CEA, CA125 and CA19-9, may be helpful in the prediction of response to systemic chemotherapy. We rationalised the following: (i) serum CEA and CA19-9 levels are elevated in over 50\% PMP patients at presentation and drop markedly following cytoreductive surgery and HIPEC (van Ruth et al, 2002); (ii) elevated CA 19-9 levels prior to cytoreductive surgery may be an independent predictor of worse progression-free survival (Baratti et al, 2007); and (iii) in a case study of a patient with DPAM treated with capecitabine, there was a decrease in CEA and CA19-9 levels (Levitz et al, 2004). Serum CA125 concentrations are commonly raised in patients with PMP, and at initial presentation, may mimic gynaecological malignancies (Pranesh et al, 2005). However, it is noteworthy that serum CA125 levels may also be raised in conditions including peritoneal irritation (Sjovall et al, 2002), pelvic inflammatory disease (Halila et al, 1986) and benign hepatic disease (Topalak et al, 2002). Although our study demonstrated elevated levels of all three markers in one-third of the cases at baseline, and reductions of $50 \%$ or more in half of these, there was no relationship between treatment response and decrease in marker level. Although the present study is relatively small, tumour marker response does not appear helpful in determining chemotherapy response.

For an unselected population of patients with PMP, over half of the cases at initial presentation will be advanced and unresectable. For these, we recommend 3-monthly CT scan. Where disease is stable, a 'watch and wait' policy with repeat imaging every 3-6 months may be prescribed. These patients should be considered for MCap chemotherapy if they become symptomatic or their disease is radiologically progressing. We recommend up to a 6-month course of treatment followed by a rest period with CT surveillance; repeat MCap courses may be prescribed if disease progresses (the maximum total dose of MMC used in this study was $42 \mathrm{mg} \mathrm{m}^{-2}$ ).

One unresolved questions from this study is that of optimising patient selection criteria. We noted that three out of the four patients that died early had aggressive histological types. Based on its effectiveness in the management of advanced colorectal cancer (Saunders and Iveson, 2006), we hypothesize that the use of oxaliplatin and 5-FU combination may be beneficial in such cases. This regimen has yet to be systematically tested and will have to 
address additional practical issues, such as the requirement for central venous access and cumulative toxicity such as parasthesia. Although the understanding of the basic biology of PMP is still in its infancy (Bibi et al, 2006), for the immediate future, systemic antitumour approaches in the treatment of advanced unresectable disease will probably emerge from existing strategies for related gastrointestinal malignancies.

\section{ACKNOWLEDGEMENTS}

We thank Mia Parkinson for her assistance with administration of the trial and data collection. MPS, ALF, NG, GW and ARG contributed to study design, data collection and clinical inter- pretation. MSW and STOD led the clinical services and contributed substantial writings on the non-surgical treatment of patients. GW undertook the quality-of-life surveys. MBT led the radiological assessment/reviews of scans. ALF, NP and SR collected and maintained the prospective database, and contributed to data upload and interpretation. RS, MPS and AGR undertook analyses and wrote early drafts. All authors contributed and approved the final manuscript.

\section{Conflict of interest}

The clinical services are supported by the National Health Service (NHS) National Commissioning Group for Highly Specialised Services. The authors state no conflicts of interest.

\section{REFERENCES}

Aaronson NK, Ahmedzai S, Bergman B, Bullinger M, Cull A, Duez NJ, Filiberti A, Flechtner H, Fleishman SB, de Haes JC et al (1993) The European Organization for Research and Treatment of Cancer QLQ-C30: a quality-of-life instrument for use in international clinical trials in oncology. J Natl Cancer Inst 85: 365-376

Baratti D, Kusamura S, Martinetti A, Seregni E, Laterza B, Oliva DG, Deraco M (2007) Prognostic value of circulating tumor markers in patients with pseudomyxoma peritonei treated with cytoreductive surgery and hyperthermic intraperitoneal chemotherapy. Ann Surg Oncol 14: 2300-2308

Bibi R, Pranesh N, Saunders MP, Wilson MS, O' Dwyer S T, Stern PL, Renehan AG (2006) A specific cadherin phenotype may characterise the disseminating yet non-metastatic behaviour of pseudomyxoma peritonei. Br J Cancer 95: 1258-1264

Borner MM, Schoffski P, de Wit R, Caponigro F, Comella G, Sulkes A, Greim G, Peters GJ, van der Born K, Wanders J, de Boer RF, Martin C Fumoleau P (2002) Patient preference and pharmacokinetics of oral modulated UFT versus intravenous fluorouracil and leucovorin: a randomised crossover trial in advanced colorectal cancer. Eur J Cancer 38: $349-358$

Crooke ST, Bradner WT (1976) Mitomycin C: a review. Cancer Treat Rev 3: $121-139$

Halila H, Stenman UH, Seppala M (1986) Ovarian cancer antigen CA 125 levels in pelvic inflammatory disease and pregnancy. Cancer 57: $1327-1329$

Hoff PM, Ansari R, Batist G, Cox J, Kocha W, Kuperminc M, Maroun J, Walde D, Weaver C, Harrison E, Burger HU, Osterwalder B, Wong AO, Wong R (2001) Comparison of oral capecitabine versus intravenous fluorouracil plus leucovorin as first-line treatment in 605 patients with metastatic colorectal cancer: results of a randomized phase III study. J Clin Oncol 19: 2282-2292

Levitz JS, Sugarbaker PH, Lichtman SM, Brun EA (2004) Unusual abdominal tumors, case 1. Pseudomyxoma peritonei: response to capecitabine. J Clin Oncol 22: 1518-1520

Moran BJ (2006) Establishment of a peritoneal malignancy treatment centre in the United Kingdom. Eur I Surg Oncol 32: 614-618

Murphy EM, Sexton R, Moran BJ (2007) Early results of surgery in 123 patients with pseudomyxoma peritonei from a perforated appendiceal neoplasm. Dis Colon Rectum 50: 37-42

NCI (2004) National Cancer Institute. Common Terminology Criteria for Adverse Events v3.0., http://www.safetyprofiler-ctep.nci.nih.gov/CTC/ CTC.aspx (accessed 27 April 2008)

Newcombe RG (1998) Two-sided confidence intervals for the single proportion: comparison of seven methods. Stat Med 17: 857-872

Ozawa S, Sugiyama Y, Mitsuhashi Y, Kobayashi T, Inaba M (1988) Cell killing action of cell cycle phase-non-specific antitumor agents is dependent on concentration-time product. Cancer Chemother Pharmacol 21: $185-190$

Padhani AR, Ollivier L (2001) The RECIST (Response Evaluation Criteria in Solid Tumors) criteria: implications for diagnostic radiologists. $\mathrm{Br}$ Radiol 74: $983-986$

Pranesh N, Menasce LP, Wilson MS, O’Dwyer ST (2005) Pseudomyxoma peritonei: unusual origin from an ovarian mature cystic teratoma. J Clin Pathol 58: $1115-1117$
Rao S, Cunningham D, Price T, Hill ME, Ross PJ, Tebbutt N, Norman AR, Oates J, Shellito P (2004) Phase II study of capecitabine and mitomycin C as first-line treatment in patients with advanced colorectal cancer. $\mathrm{Br}$ J Cancer 91: 839-843

Renehan AG, O'Dwyer ST, Stern PL (2008) Pseudomyxoma peritonei In Cancer Encyclopedia, Schwab M (ed). London: Spinger

Ronnett BM, Shmookler BM, Sugarbaker PH, Kurman RJ (1997) Pseudomyxoma peritonei: new concepts in diagnosis, origin, nomenclature, and relationship to mucinous borderline (low malignant potential) tumors of the ovary. Anat Pathol 2: 197-226

Rout S, Renehan AG, Parkinson M, Saunders MP, Fulford PE, Wilson MS, O' Dwyer ST (2008) The treatments and outcomes of peritoneal tumours through a centralised national service (UK). Dis Colon Rectum (in press)

Saunders M, Iveson T (2006) Management of advanced colorectal cancer: state of the art. Br J Cancer 95: 131-138

Sjovall K, Nilsson B, Einhorn N (2002) The significance of serum CA 125 elevation in malignant and nonmalignant diseases. Gynecol Oncol 85: $175-178$

Smeenk RM, Verwaal VJ, Zoetmulder FA (2007) Learning curve of combined modality treatment in peritoneal surface disease. Br J Surg 94: $1408-1414$

Sprangers MA, te Velde A, Aaronson NK (1999) The construction and testing of the EORTC colorectal cancer-specific quality of life questionnaire module (QLQ-CR38). European Organization for Research and Treatment of Cancer Study Group on quality of life. Eur J Cancer 35: $238-247$

Sugarbaker PH (2006) New standard of care for appendiceal epithelial neoplasms and pseudomyxoma peritonei syndrome? Lancet Oncol 7: $69-76$

Sugarbaker PH, Chang D (1999) Results of treatment of 385 patients with peritoneal surface spread of appendiceal malignancy. Ann Surg Oncol 6 : $727-731$

Sulkin TV, O'Neill H, Amin AI, Moran B (2002) CT in pseudomyxoma peritonei: a review of 17 cases. Clin Radiol 57: 608-613

Topalak O, Saygili U, Soyturk M, Karaca N, Batur Y, Uslu T, Erten O (2002) Serum, pleural effusion, and ascites CA-125 levels in ovarian cancer and nonovarian benign and malignant diseases: a comparative study. Gynecol Oncol 85: $108-113$

Van Cutsem E, Twelves C, Cassidy J, Allman D, Bajetta E, Boyer M, Bugat R, Findlay $M$, Frings $S$, Jahn $M$, McKendrick J, Osterwalder $B$, Perez-Manga G, Rosso R, Rougier P, Schmiegel WH, Seitz JF, Thompson P, Vieitez JM, Weitzel C, Harper P (2001) Oral capecitabine compared with intravenous fluorouracil plus leucovorin in patients with metastatic colorectal cancer: results of a large phase III study. J Clin Oncol 19: 4097-4106

van Ruth S, Hart AA, Bonfrer JM, Verwaal VJ, Zoetmulder FA (2002) Prognostic value of baseline and serial carcinoembryonic antigen and carbohydrate antigen 19.9 measurements in patients with pseudomyxoma peritonei treated with cytoreduction and hyperthermic intraperitoneal chemotherapy. Ann Surg Oncol 9: 961-967

Witkamp AJ, de Bree E, Kaag MM, van Slooten GW, van Coevorden F, Zoetmulder FA (2001) Extensive surgical cytoreduction and intraoperative hyperthermic intraperitoneal chemotherapy in patients with pseudomyxoma peritonei. Br J Surg 88: 458-463 